\title{
A time resolved VUV fluorescence study of hydrogen clusters: evidence of a liquid phase
}

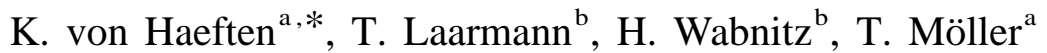 \\ ${ }^{a}$ Hamburger Synchrotronstrahlungslabor HASYLAB at Deutsches Elektronen Synchrotron DESY, Notkestr. 85, 22603 Hamburg, \\ Germany \\ ${ }^{\mathrm{b}}$ II Institut für Experimentalphysik, Universität Hamburg, Germany
}

\begin{abstract}
The mobility of $\mathrm{Xe}$ atoms captured from a cross jet by hydrogen clusters is investigated with time resolved VUV fluorescence excitation spectroscopy. A band blue shifted by $0.5 \mathrm{eV}$ with respect to the $5 \mathrm{p} \rightarrow 6 \mathrm{~s}$ transition of free $\mathrm{Xe}$ atoms is observed. It is attributed to Xe atoms profoundly embedded in hydrogen clusters and surrounded by several layers of hydrogen. The complete absence of features related to surface sites gives evidence that after the pick up process the xenon atoms penetrate deep into the interior of the hydrogen clusters. This indicates that hydrogen clusters show a liquid-like behavior similar to helium clusters, though their estimated temperature $(\sim 6 \mathrm{~K})$ is much below the triple point of hydrogen (14 K). (C) 2000 Elsevier Science B.V. All rights reserved.
\end{abstract}

Keywords: Thermodynamic phase of clusters; Hydrogen; Liquid; Mobility; VUV; Spectroscopy; Fluorescence

\section{Introduction}

Clusters are well known to have a significantly lower melting temperature than their bulk counterparts due to their small size. Presently, the size dependence of thermodynamic properties of clusters are widely studied [1]. However, macroscopic properties like liquid and solid begin to lose their meaning when the size of a substance is reduced to some 10 to 1000 atoms. Particular consideration of the method which should be applied to investigate whether a cluster is liquid or solid is therefore required. Calculations show for example that in some

*Corresponding author.

E-mail address: haeften@desy.de (K. von Haeften) cases particles in the cluster show an increased tendency to diffuse [2]. This is related to a liquid phase, while a nearly constant mean square displacement belongs to a solid phase. Additionally, calculated density profiles or mean square fluctuations yield information whether a cluster is more solid or liquid like [3]. The mobility of an impurity particle in the cluster is affected in a similar way by the phase of the cluster. Measuring the mobility is therefore a method to ascertain the phase of clusters.

In the case of hydrogen the phase of clusters is of considerable interest, because cluster beams have been suggested for the production of ultra cold hydrogen, far below the melting temperature, but still being liquid. This is the prior condition to study possible superfluid hydrogen. Para-hydrogen $(J=0)$ 
has been predicted to undergo Bose-Einstein condensation (BEC) and to exhibit a superfluid phase [4], provided it is still liquid at the phase transition temperature. According to the theoretical predictions of Ginzburg and Sobyanin, the superfluid phase transition temperature of hydrogen should lie around 4-5 K [4]. Normal hydrogen is solid at this temperature, because the triple point is at $14 \mathrm{~K}$. Many attempts have been undertaken to under cool hydrogen $[5,6]$. Small droplets of hydrogen with diameters ranging from 30 to $1000 \mu \mathrm{m}$ have been levitated in pressurized helium, but these rather large droplets remained liquid only above $10 \mathrm{~K}$ [7].

As stated above, a promising attempt to circumvent solidification is the application of cluster beam techniques. Clusters, prepared in a nozzle expansion, are created in a region located $\sim 3$ to 10 times of the nozzle diameter downstream from the nozzle. As condensation into clusters starts, the clusters are supposed to be overheated. They fly without any interaction through the vacuum and cool themselves by evaporation of particles. Especially for hydrogen this cooling mechanism is very efficient, because the molecules are relatively loosely bound. All this happens on a time scale of micro- to milliseconds, depending on the experimental set up that is used. The flight time of the clusters can therefore be sufficiently below the calculated time that is needed for the droplets to become eventually solid [6]. Furthermore, by cooling themselves the clusters in a beam gain very low temperatures. Knuth et al. reported calculations and experiments to produce super cooled hydrogen droplets using supersonic beam methods [5] and concluded that under certain conditions such as coexpansion with helium gas a temperature of 4-5 $\mathrm{K}$ of the droplets can be attained. However, they could not prove experimentally that hydrogen clusters are liquid and pointed out that this had conclusively to be shown later.

While $\mathrm{SF}_{6}$-doped hydrogen clusters have already been investigated experimentally [8], indications for possible superfluidity have so far only been discussed in theoretical studies [3,9,10]. Superfluidity seems to occur only for small pure para-hydrogen containing less than 20 molecules and, in contrast to the predictions by Ginzburg and Sobyanin, only at temperatures below $2 \mathrm{~K}[3,9,10]$. However, if hydrogen clusters which had a solid core but a liquid surface layer exist, they would be quite interesting in terms of BEC, because (i) the surface has a considerable lower density $[3,10]$, which is desirable to push up the superfluid phase transition temperature, or (ii) they may reveal a superfluid surface which is restricted to two dimensions. Superfluidity of $\mathrm{H}_{2}$ films which have been doped with alkali metal atoms have recently been the subject of path integral Monte Carlo calculations [11]. While experiments show that pure hydrogen layers have a triple point of $5.8 \mathrm{~K}$ [12], the calculations predict, that the alkali-doped hydrogen layer could be prevented to become solid, if the alkali impurities had been introduced at the right concentration, and a superfluid phase transition should be observable at temperatures below $1.2 \mathrm{~K}$.

\section{Experiment}

\subsection{Method}

Here we report on an experimental study in order to check whether $\mathrm{H}_{2}$ clusters are liquid or solid. The experiment works in principle as follows: free clusters of normal-hydrogen ( $25 \%$ para- and $75 \%$ ortho-) are created by supersonic expansion of hydrogen gas and fly through vacuum. They pick up xenon atoms from a cross beam and then intersect with a synchrotron radiation light beam (Fig. 1). Xe atoms embedded in the cluster are investigated with fluorescence excitation spectroscopy. The fluorescence excitation spectra contain information, where the Xe

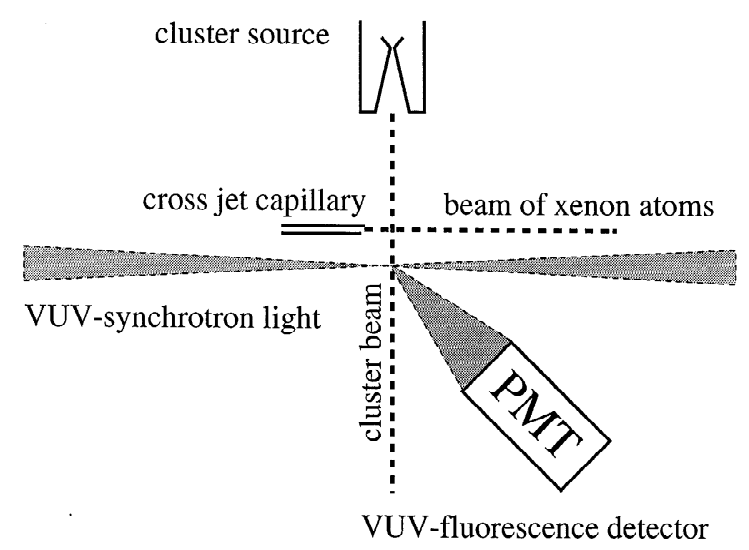

Fig. 1. Schematic sketch of the experimental set-up. 
atom is located in the cluster. From other experiments we know that atoms stick to the surface if the cluster is solid, while they penetrate into the interior region if it is liquid [13,14]. This holds only, if the interaction between guest and host is stronger than the host-host interaction. For $\mathrm{Xe} / \mathrm{H}_{2}$ this is certainly fulfilled.

This experimental method works well for van der Waals systems and has recently been tested for all rare gas clusters $[13,14]$. The preferred site of the xenon atom is correlated to the expected thermodynamic phase of the cluster. For example, in helium clusters, which are definitely liquid, only interior sites have been observed, while in argon clusters, which are solid, only surface sites are found under pick up conditions. An interesting intermediate case are neon clusters, which exhibit interior sites up to a cluster size of $N=200$ and surface sites for the larger clusters. Small neon clusters seem to be liquid while larger ones become solid [14]. On the other hand, doped solid clusters (like argon) that are produced in a coexpansion of a mixture of the desired gas compounds exhibit both interior and surface sites above a threshold of the size of roughly 50 atoms. If experimentally applicable the coexpansion method can hence be used to distinguish between both sites. For hydrogen, this is unfortunately not possible, because the $\mathrm{Xe}$ atoms in the gas mixture would freeze and clog the nozzle, which has a temperature around $30 \mathrm{~K}$ in order to produce hydrogen clusters.

Discrete impurity sites are related to well separated absorption bands (Fig. 2) due to excited states that can be explained in terms of a model of perturbed excited atoms $[15,16]$. They can be identified [17] with the aid of luminescence excitation spectroscopy. The bands are blue-shifted with respect to the first atomic resonance line of xenon. The excited state of the impurity atom is perturbed in the following way: the repulsive force between the electronically excited Rydberg orbital of the Xeimpurity atom and the surrounding closed shell atoms or molecules of the cluster pushes the latter away leading to a minimum of the potential located at a larger intermolecular distance than for the ground state [18]. Therefore, optical transitions take place between the minimum of the ground state potential and the repulsive part of the potential curve of the excited state (see Fig. 2). The strength of the blue shift is related to the coordination number of the xenon atoms. Xe atoms on the surface, which are not completely surrounded by cluster atoms, exhibit therefore a smaller blue shift with respect to those at interior sites.

The results of the extensive study of rare gas clusters permits much confidence both in the inter-
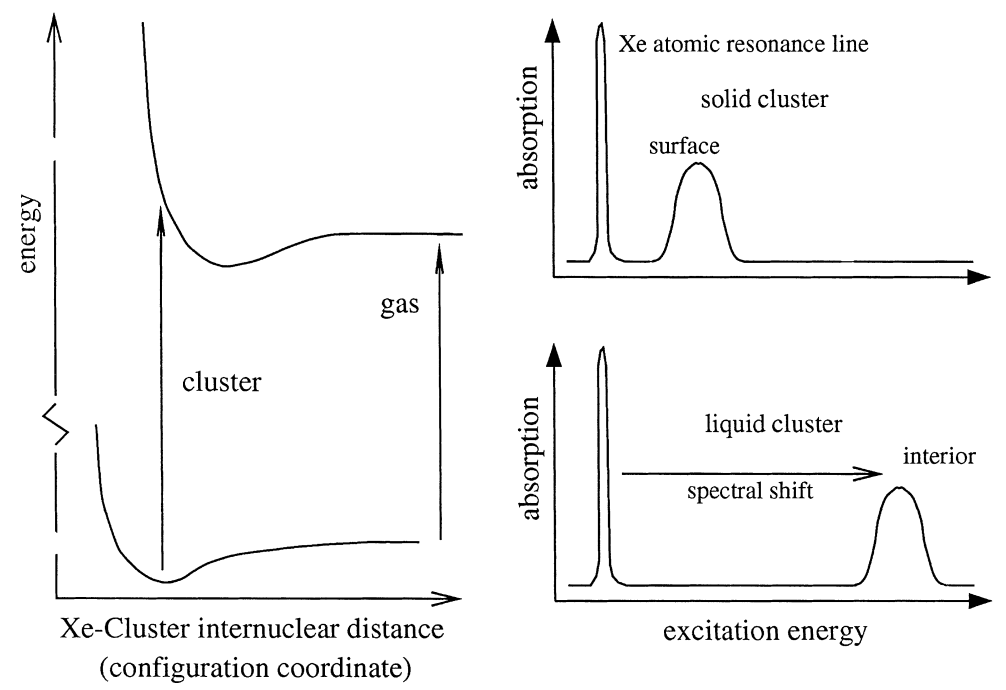

Fig. 2. Schematic illustration of the origin of the blue-shifted bands related to impurity sites in van der Waals clusters. The magnitude of the blue shift is connected to the coordination number of the impurity. 
pretation of the spectra and in the attribution to the phase of the clusters $[13,16]$. We believe therefore, that different thermodynamic phases of clusters can be distinguished in this way.

\subsection{Experimental set-up}

The experiments have been carried out at the experimental station $C L U L U$ at the synchrotron light source DORIS at HASYLAB (Fig. 1). A detailed description of the experiment is given in Ref. [19]. Briefly, hydrogen clusters are produced in a supersonic expansion covering a size range up to $10^{5}$ molecules. The mean cluster size depends on expansion parameters such as nozzle diameter, temperature and pressure of the gas. Several flat orifices of 40 $\mu \mathrm{m}, 60 \mu \mathrm{m}$ and a conical nozzle $\left(2 \phi=8^{\circ}\right)$ of 200 $\mu \mathrm{m}$ in diameter were used. Spectra obtained with different nozzle geometries revealed very similar experimental results. In the following we refer therefore to experiments where the conical nozzle has been applied, because it provided the best signal to noise ratio. For the largest hydrogen clusters a stagnation pressure $P_{0}$ of 270 mbar and a nozzle temperature $T_{0}$ of $26 \mathrm{~K}$ have been used. These parameters lead to an average cluster size of 225000 molecules according to a scaling law from Obert, who investigated the size of hydrogen clusters under similar experimental conditions [20]. Ten mm downstream from the nozzle the hydrogen clusters picked up xenon atoms from a cross jet. The cross jet nozzle, a thin capillary of $400 \mu \mathrm{m}$ in diameter (injection needle), was placed $2.5 \mathrm{~mm}$ away from the cluster beam axis to avoid any disturbance of the cluster beam and to obtain the highest possible signal. The pressure of the xenon gas before the capillary ranged from 7 to 30 mbar. One $\mathrm{mm}$ further downstream the doped clusters reached the focus of the monochromatized synchrotron light. The impurity xenon atoms were excited in an energy range between 8 and $10 \mathrm{eV}$ with a spectral resolution of 2.5 $\AA$ (12 meV). By measuring the fluorescence yield as a function of the excitation energy using a solar blind photo multiplier tube (Hamamatsu R2050) the absorption of the doped clusters has been monitored. The absorption is usually proportional to the fluorescence yield, if no quenching occurs [19]. This assumption is well fulfilled in the case of Xe-doped hydrogen clusters, because the exited states of the impurity atom lay within the band gap of hydrogen. Time correlated excitation spectra with respect to different fluorescence life times can be obtained due to the pulsed nature of synchrotron light. The separation between the light pulses is $200 \mathrm{~ns}$, while the pulse duration is $130 \mathrm{ps}$. This technique allows us to improve the signal to noise ratio, and in addition, to distinguish between different absorption features, as shown later.

\section{Results and discussion}

Fig. 3 shows a fluorescence excitation spectrum of large size xenon-doped hydrogen clusters containing 65000 molecules $\left(P_{0}=92 \mathrm{mbar}, T_{0}=28.5 \mathrm{~K}\right)$. Only short-lived-fluorescence has been recorded within a time range from 0 to $15 \mathrm{~ns}$ after excitation. This results in an increased signal to noise ratio, because the radiative life time of single impurity $\mathrm{Xe}$ atoms is a few nanoseconds. Apart from the first $\mathrm{Xe}$ atomic resonance line $5 \mathrm{p} \rightarrow{ }^{2} \mathrm{P}_{3 / 2} 6 \mathrm{~s}[3 / 2]_{1}$ at $8.437 \mathrm{eV}$, which is due to background gas, a broad absorption band peaking at a photon energy of $8.94 \mathrm{eV}$ is observed.

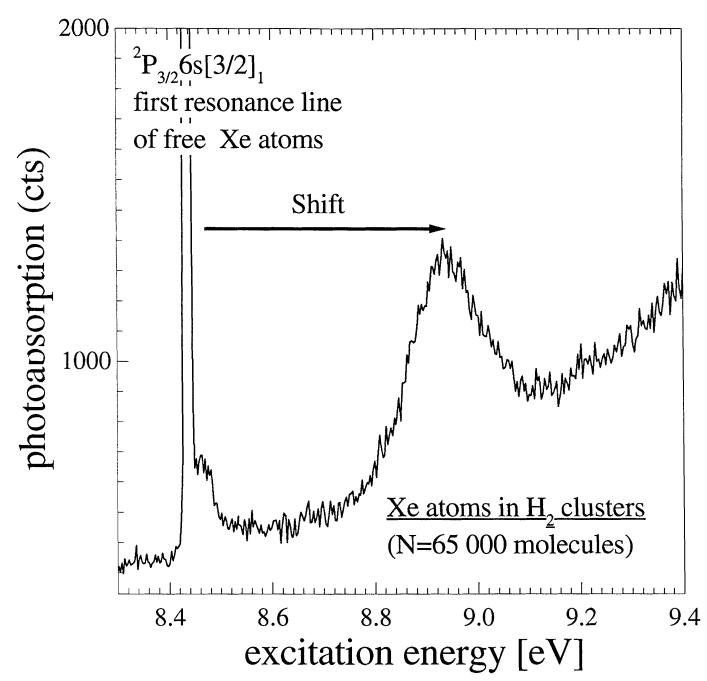

Fig. 3. Fluorescence excitation spectrum of Xe-doped $\mathrm{H}_{2}$ clusters containing 65000 molecules. The band at $8.94 \mathrm{eV}$ is due to $\mathrm{Xe}$ atoms embedded in the interior of $\mathrm{H}_{2}$ clusters. The weak features at $8.47 \mathrm{eV}$ is related to small $\mathrm{Xe}-\mathrm{H}_{2}$ complexes which are due to scattering with the background gas. The rising background signal results from Mie scattering of the large clusters. 
This band can be attributed to an excitation of Xe atoms surrounded by several $\mathrm{H}_{2}$ molecules, very similar to the absorption features of $\mathrm{Xe}$ atoms embedded in He. A similar band can also be observed for the other Xe-doped rare gas clusters, if they are produced by coexpansion techniques [13]. The band appears at cross jet pressures larger than 7 mbar for all the clusters studied in this work ranging in size from 1000 to 225000 molecules. For clusters up to a size of 65000 molecules the intensity is nearly proportional to the cross jet pressure, provided it does not not exceed values higher than 18 mbar. Small deviations from the linearity at very low pressures are presumably due to small contributions from the absorption of Xe dimers located at interior sites.

The strong blue shift of $0.5 \mathrm{eV}$ with respect to the resonance line of the free $\mathrm{Xe}$ atom indicates that the coordination number is rather high and that the atom is surrounded by at least two layers of molecules [16]. Spectral shifts of surface sites are two to three times smaller in all van der Waals systems investigated so far [13]. The blue shift observed here is in the same order as the one of xenon atoms embedded in liquid helium clusters, which is $0.4 \mathrm{eV}$ [13]. Moreover, the width of the band (162 meV (FWHM)) is comparable to that of Xe-doped helium clusters (195 meV (FWHM)). In general, a tendency is observed, that the width of impurity absorption bands becomes broader the lighter the constituents of the clusters are. These findings may be related to the increased zero point motion of the impurity in the cluster.

The weak features at $8.47 \mathrm{eV}$, close to the $\mathrm{Xe}$ resonance line, are probably related to small $\mathrm{Xe}-\mathrm{H}_{2}$ complexes which are due to scattering with the background gas. The background signal continuously rising towards higher excitation energy results from Mie scattering of large $\mathrm{H}_{2}$ clusters.

It should be pointed out that the intensity of the fluorescence band of $\mathrm{Xe}$ atoms in $\mathrm{H}_{2}$-clusters is comparable to that of Xe-doped He-clusters produced under similar conditions. We conclude therefore that xenon is completely solved in $\mathrm{H}_{2}$-clusters as it is the case for He-clusters.

Fig. 4 shows time correlated spectra of Xe-doped hydrogen clusters of two different sizes (65000 and 225000 molecules) and cross jet pressures (7 mbar and 24 mbar). For each cluster size three different spectra display the fluorescence yield within time ranges from 0 to $15 \mathrm{~ns}$, from 15 to $100 \mathrm{~ns}$ and the total fluorescence yield. In the case of the larger clusters and the high cross jet pressure it is observed that the features at energies around $8.9 \mathrm{eV}$ recorded within the short time-window and the long timewindow are slightly shifted: the maximum of the band of the first spectrum is found at $8.94 \mathrm{eV}$, while the maximum of the spectrum which belongs to the long time-window is at $8.92 \mathrm{eV}$. The latter can be attributed to dimers. Such a red shift of the absorption band of the $\mathrm{Xe}$ dimer with respect to the monomer is reported in a molecular dynamics simulation for argon clusters doped with xenon atoms and dimers [21]. Furthermore, the life time of $\sim 100 \mathrm{~ns}$ is characteristic for Xe dimers [22]. Corresponding to the rather large blue shift with respect to the resonance line of the free $\mathrm{Xe}$ atom it is conclusive that they are formed inside and reside at an interior site of the $\mathrm{H}_{2}$-cluster. The formation of Xe dimers with their characteristic rather long radiative life time of $100 \mathrm{~ns}$ has also been found for small Xe-doped neon and helium clusters [13,14]. Moreover, the formation of dimers is reflected by the ratio of the intensities of the two bands as a function of increasing cluster size and cross jet pressure, which can be seen in Fig. 4. If the intensities of each band of the spectrum of the 225000 -molecule large clusters is divided by the intensities of the same peaks of the spectrum which belongs to the 65000 -molecule large clusters, it is observed that the intensity ratio of the dimer band is 3.14 , compared to 2.5 for the monomer, while the intensity of the total fluorescence yield is nearly proportional to the cross jet pressure. That means, that as expected with increasing cross jet pressure and cluster size the clusters pick up more atoms which coagulate more likely into dimers. This process is well known from investigations of doped helium clusters [23] and typical for liquid clusters.

The fluorescence excitation spectrum of the long lived states exhibits also features at energies between the bands discussed above and the resonance line of free xenon atoms. These features are nearly absent for the smallest hydrogen clusters that have been investigated in this work and become stronger the larger the cluster size and the higher the cross jet 


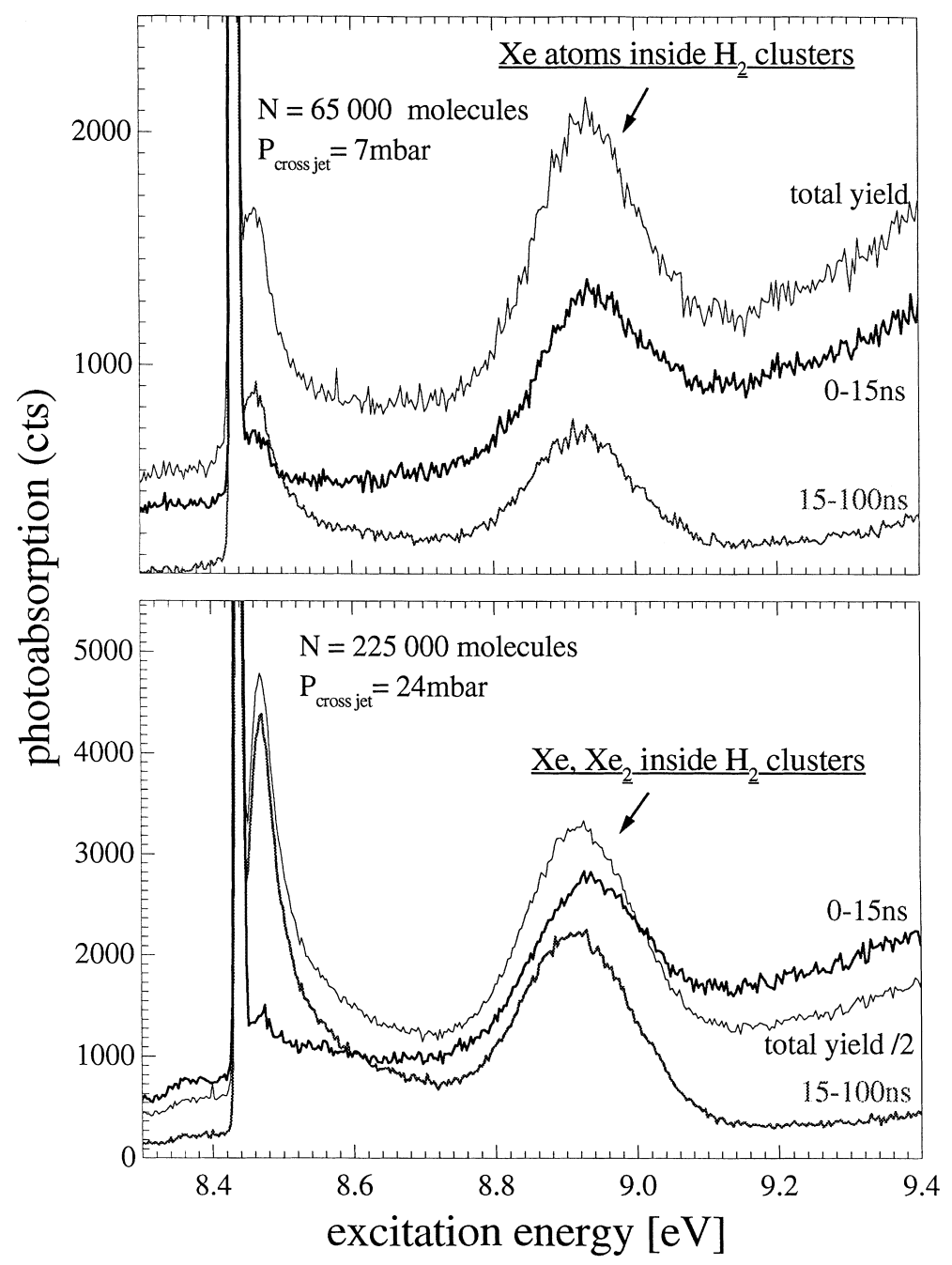

Fig. 4. Time correlated fluorescence excitation spectra. The fluorescence band at $8.9 \mathrm{eV}$ characterized by a short decay time is due to monomers, while the long-lived-fluorescence yield is due to dimers inside hydrogen clusters. Increasing cluster size and cross jet pressure lead to a higher pick up rate of Xe atoms which coagulate into dimers.

pressure is (Fig. 4). Especially the peaks at $8.46 \mathrm{eV}$ become stronger than the dimer band. The features seem to be unique for hydrogen and have not been observed for rare gas clusters. Most similarities regarding the shape of the features are found in the case of small neon clusters $(n=8)$ doped with Xe atoms by coexpansion technique. However, the width of the peak at $8.46 \mathrm{eV}$ is much narrower for neon. Although we cannot give a conclusive interpretation, it is very unlikely that surface sites are responsible for these features for two reasons: (i) surface sites should become less prominent with increasing cluster size and with cross jet pressure. (ii) The long decay time of the emitting states gives evidence that at least two Xe atoms are involved.

Presumably, the features are due to small $\mathrm{Xe}-\mathrm{H}_{2}$ aggregates, containing up to a few Xe atoms covered by less than one shell of $\mathrm{H}_{2}$ molecules. This agrees with the following experimental manifestations: (i) the strength of the blue shift indicates that the Xe complexes are not free, but attached to at least some hydrogen molecules. (ii) The similarities to neon 
mentioned above suggest that the size of the complexes is rather small. Therefore we attribute the features to the absorption of small $\mathrm{Xe}-\mathrm{H}_{2}$ aggregates, which are either due to the fraction of small hydrogen clusters which always accompany the clusters in a beam that is characterized by a rather large mean size, or, more plausible, to aggregates that are expelled from $\mathrm{H}_{2}$ clusters, when too much heat of condensation of the small Xe-aggregates is dissipated into the $\mathrm{H}_{2}$ cluster.

Summarizing the experimental results keeping in mind the findings for Xe-doped rare gas clusters a liquid phase of small hydrogen clusters is favorable. Independent of the cluster size, single Xe atoms and dimers at the interior are observed. These findings are characteristic for liquid clusters like helium and not observed for solid clusters like Ar or $\mathrm{Kr}$. On the other hand, there are some differences to helium. Helium clusters that are highly doped with xenon atoms do not simultaneously reveal spectral bands related to interior sites and $\mathrm{Xe}_{n}-\mathrm{He}_{m}$ aggregates [13]. On the basis of our results we can therefore not exclude that the clusters have a solid core as predicted by a recent theoretical study [2]. In this work, the quantum dynamics of Li-doped and pure para-hydrogen clusters up to a size of 180 molecules has been investigated. Though the doping of $\mathrm{Li}$, which resides at the cluster surface, cannot directly be compared with the doping of Xe, some remarkable results of this work agree qualitatively with our findings. Clusters of the size of $n=55$ are liquid at $4.0 \mathrm{~K}$ while at the same temperature the $n=180$ clusters have a solid core and a liquid surface region. The diffusive and vibrational properties of the cluster do not seem to be affected by the Li impurity. In general, hydrogen clusters have a tendency to melt at the surface [2]. These results manifest the substantial drop of the melting temperature by reducing the size of the cluster and agree almost with our interpretation of hydrogen clusters having at least a liquid surface and maybe a solid core.

\section{Conclusion}

The mobility of Xe atoms in hydrogen clusters with its implications to the thermodynamic properties of free hydrogen clusters have been investigated by measuring fluorescence excitation spectra of Xe atoms captured by large $\mathrm{H}_{2}$ clusters. The large spectral shift of $0.5 \mathrm{eV}$ of the absorption band with respect to the resonance line of free xenon atoms indicates that the Xe atoms are deep in the interior of the cluster. Surface sites, as observed for solid clusters like Ar have not been found. These findings and the formation of dimers in the interior of the clusters at moderate pick up densities give evidence that $\mathrm{H}_{2}$-clusters ( $\mathrm{N}$ up to 225000 ) are liquid, at least close to the surface. From the comparison of the spectral features with those of Xe-doped helium clusters and their dependence on the pick up pressure it is concluded that larger clusters may have a solid core, as predicted from theory [2].

\section{Acknowledgements}

The authors are grateful to Prof. J. Jortner for communicating unpublished results for $\mathrm{Ar}_{n} \mathrm{Xe}_{2}$ clusters.

\section{References}

[1] R.S. Berry, Adv. Chem. Phys. 70 (1988) 74.

[2] K. Kinugawa, P.B. Moore, M. Klein, J. Chem. Phys. 106 (3) (1996) 1154.

[3] K.B. Whaley, Structure and dynamics of quantum clusters, Int. Rev. Phys. Chem. 13 (1994) 41-84.

[4] V.I. Ginzburg, A.A. Sobyanin, JETP 15 (1972) 242-244.

[5] E.L. Knuth, G.M. Schünemann, J.P. Toennies, J. Chem. Phys. 102 (15) (1995) 6258.

[6] H.J. Maris, G.M. Seidel, T.E. Huber, J. Low. Temp. Phys. 51 (1983) 471, and references therein.

[7] H.J. Maris, G.M. Seidel, F.I.B. Williams, Phys. Rev. B 36 (1987) 6799.

[8] S. Goyal, D.L. Schutt, G. Scoles, Chem. Phys. Lett. 196 (1.2) (1992) 123.

[9] P. Sindzingre, D.M. Ceperley, M.L. Klein, Phys. Rev. Lett. 67 (14) (1991) 1871.

[10] M.A. McMahon, K.B. Whaley, Chem. Phys. 182 (1994) 119.

[11] M.C. Gordillo, D.M. Ceperley, Phys. Rev. Lett. 79 (16) (1997) 3010.

[12] F.C. Lui, Y.M. Lui, O.E. Vilches, Phys. Rev. B 51 (1995) 2848.

[13] R. von Pietrowski, Spektroskopische Untersuchungen an edelgasdotierten Edelgasclustern, thesis Fachbereich Physik, Universität Hamburg, 1997.

[14] R. von Pietrowski, M. Rutzen, K. von Haeften, S. Kakar, T. Möller, Z. Phys. D 40 (1997) 22. 
[15] M. Lengen, M. Joppien, R. von Pietrowski, J. Wörmer, T. Möller, Chem. Phys. Lett. 229 (1994) 362.

[16] A. Goldberg, A. Heidenreich, J. Jortner, J. Phys. Chem. 99 (9) (1995) 2662.

[17] M. Lengen, M. Joppien, R. Müller, J. Wörmer, T. Möller, Phys. Rev. Lett. 68 (1992) 2362.

[18] I. Messing, B. Raz, J. Jortner, J. Chem. Phys. 66 (15) (1977) 2239.

[19] R. Karnbach, M. Joppien, J. Stapelfeldt, J. Wörmer, T. Möller, Rev. Sci. Instrum. 64 (1993) 2838-2849.
[20] W. Obert, International Symposium on Rarefied Gas Dynamics, Cannes, July, 3-8, 1978.

[21] J. Jortner, private communication.

[22] J. Stapelfeldt, Ein neues Experiment für Fluoreszenzuntersuchungen an Edelgas-Clustern vom Dimer bis zum Mikrokristall, thesis Fachbereich Physik, Universität Hamburg, 1990.

[23] M. Lewerenz, B. Schilling, J.P. Toennies, J. Chem. Phys. 102 (20) (1995) 8191. 